Cite this: Phys. Chem. Chem. Phys. 2013 15, 2466

Received 30th August 2012, Accepted 3rd December 2012 DOI: $10.1039 / c 2$ cp43036c www.rsc.org/pccp

\title{
Proton conductivity of columnar ceria thin-films grown by chemical vapor deposition
}

\author{
Tae-Sik Oh, $\dagger^{\mathrm{a}}$ David A. Boyd, ${ }^{\mathrm{b}}$ David G. Goodwin ${ }^{\mathrm{b}}$ and Sossina M. Haile*ac
}

\begin{abstract}
Columnar thin films of undoped ceria were grown by metal-organic chemical vapor deposition. The films, deposited on Pt-coated $\mathrm{MgO}(100)$ substrates, display a columnar microstructure with nanometer scale grain size and $\sim 30 \%$ overall porosity. Through-plane (thickness mode) electrical conductivity was measured by AC impedance spectroscopy. Proton conduction is observed below $350-400{ }^{\circ} \mathrm{C}$, with a magnitude that depends on gas-phase water vapor pressure. The overall behavior suggests proton transport that occurs along exposed grain surfaces and parallel grain boundaries. No impedance due to grain boundaries normal to the direction of transport is observed. The proton conductivity in the temperature range of $200-400{ }^{\circ} \mathrm{C}$ is approximately four times greater than that of nanograined bulk ceria, consistent with enhanced transport along aligned grain surfaces in the CVD films.
\end{abstract}

\section{Introduction}

Acceptor doped ceria is a well known oxygen ion conductor that can function as an electrolyte in a solid oxide fuel cell in the intermediate temperature range. ${ }^{1}$ In recent years it has been observed that, upon nanostructuring, ceria can display significant proton conductivity at much lower temperatures than that required to activate oxide ion motion. The connection between grain size and magnitude of the proton conductivity in the nanostructured materials displaying this unusual phenomenon, as well as the observation that proton uptake and transport in bulk, microcrystalline ceria are negligible, ${ }^{2-4}$ has led to the general consensus that the proton conduction occurs through a grain boundary (or surface) mediated process. ${ }^{5,6}$ This insight suggests that a material with an aligned microstructure, with grain boundaries and/or open pore channels parallel to the path of proton transport, may lead to proton conductivities that are even larger than those reported to date. Accordingly, we have prepared in this work thin-film ceria with columnar grains and characterized the conductivity along the film growth direction. In addition to the creation of structures with enhanced conductivity, the investigation sheds some new light on the proton transport mechanism.

\footnotetext{
${ }^{a}$ Materials Science, California Institute of Technology, Pasadena, CA, USA. E-mail: smhaile@caltech.edu; Fax: +1 626395 3933; Tel: +1 6263952958 ${ }^{b}$ Mechanical Engineering, California Institute of Technology, Pasadena, CA, USA ${ }^{c}$ Chemical Engineering, California Institute of Technology, Pasadena, CA, USA $\dagger$ Current address: Department of Chemical and Biomolecular Engineering, University of Pennsylvania, Philadelphia 19014, USA.
}

\section{Experimental procedures}

Undoped ceria films were grown by chemical vapor deposition (CVD) using an in-house constructed, cold-walled vertical reactor. ${ }^{7}$ Dopants were not utilized in order to avoid possible composition gradients in the as-grown films. To facilitate through-plane (or thickness mode) conductivity measurements, deposition was carried out on $\mathrm{MgO}(100)$ substrates (MTI crystals) first coated with Pt $(\sim 200 \mathrm{~nm})$ by DC sputter deposition (AJA International ATC Orion system, Ar 3 mTorr, $10 \mathrm{sccm}$, ambient temperature). The cerium precursor for CVD film growth was the commercially available compound Ce(tmhd) ${ }_{4}$ (Strem CAS\#: 18960-54-8, 99.9\% cation purity). The as-received solid precursor was used without further purification and delivered to the reaction chamber following a procedure described elsewhere. ${ }^{8}$ The deposition conditions are provided in detail in Table 1. Top Pt electrodes were applied to the ceria films by DC sputtering (as above). It was observed that a minimum Pt thickness of $50 \mathrm{~nm}$ was required to prevent film dewetting and enable reliable measurements. A shadow mask was used to limit electrode deposition to circular regions with a nominal diameter of 1.5 to $3 \mathrm{~mm}$. Doing so prevented short

Table 1 Film growth conditions for chemical vapor deposition of undoped ceria

\begin{tabular}{ll}
\hline Parameter & Value \\
\hline Growth temperature $\left({ }^{\circ} \mathrm{C}\right)$ & 500 \\
Growth pressure (Torr) & 3 \\
Growth time (min) & $240-270$ \\
Gas flow (sccm) & Argon : 100, $\mathrm{O}_{2}: 50$ \\
Ce precursor evaporation temperature $\left({ }^{\circ} \mathrm{C}\right)$ & 190
\end{tabular}



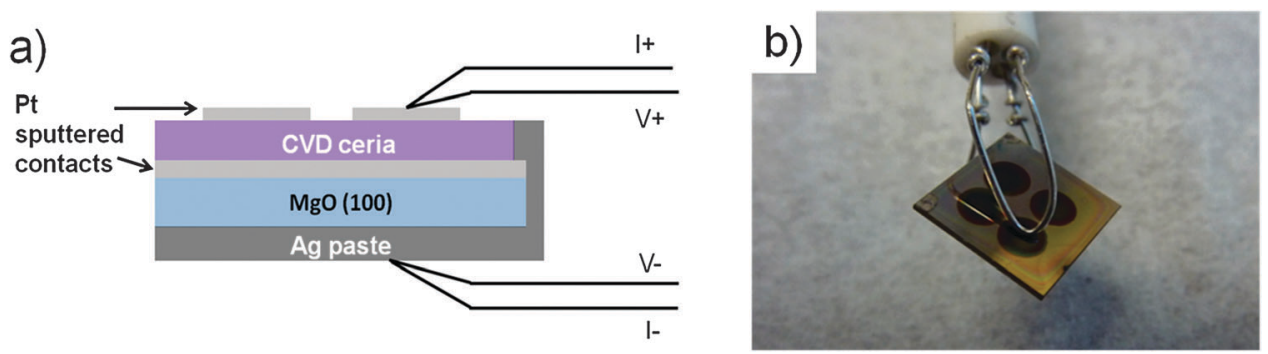

Fig. 1 Through-plane (or thickness-mode) conductivity measurement configuration: (a) schematic diagram (dimensions not to scale), and (b) optical image of the sample loaded into the test fixture. Ceria films were deposited on platinum coated $\mathrm{MgO}(100)$ substrates. Silver paste provided contact to the $200 \mathrm{~nm}$ thick Pt bottom electrode. Spring-loaded Pt wires provided contact to the 50-250 nm thick top Pt electrode.

circuits from forming at the film edges, and also enabled, by application of multiple isolated top electrodes, multiple conductivity measurements from a single film. A schematic of the measurement configuration is presented in Fig. 1.

Scanning electron microscopy (SEM) and X-ray diffraction (XRD) measurements were performed on the as-deposited films using a Carl Zeiss LEO 1550VP and a Panalytical X'pert pro MRD system ( $45 \mathrm{kV}, 40 \mathrm{~mA})$, respectively. The grain size was estimated from the XRD peak breadth using the Scherrer equation $^{9}$ after accounting for instrumental peak broadening using a YSZ single crystal and data collected under identical conditions. The microstructural features and phase behavior were reproducibly observed in many different films and only representative results are reported.

Film conductivity was measured by AC impedance spectroscopy. Samples of the configuration shown in Fig. 1 were placed in a vertical tube furnace equipped with a gas control. The gaseous environment was either (i) high-purity oxygen passed through molecular sieves to remove residual water, or (ii) the same oxygen gas humidified to a specified level. In the latter case, the $p \mathrm{H}_{2} \mathrm{O}$ was set by saturating the incoming oxygen gas with water held at a specified temperature. This humidified stream was then either mixed with dry oxygen gas to reach an even lower water level or used directly without dilution. The quoted water partial pressures correspond to the expected values without further experimental confirmation. Measurements were carried out by first heating the samples to the highest temperature examined $\left(500{ }^{\circ} \mathrm{C}\right)$ under the gaseous environment of interest. A $5 \mathrm{~h}$ dwell at this temperature allowed for stabilization of the electrical contacts and equilibration of the ceria film with the gas phase. Measurements were performed at several different temperatures upon step-wise cooling from this equilibration condition. The ramp rate was $\leq 2$ degrees per minute to minimize possible thermal shock, and the dwell time under each measurement condition was $\geq 30 \mathrm{~min}$. Measurements were limited to a maximum temperature of $500{ }^{\circ} \mathrm{C}$, equal to the film growth temperature, to preclude the possibility of evolution of the film microstructure over the course of the data collection. The stability of the Pt film top-electrodes was confirmed by post-measurement SEM imaging. Impedance data were collected using a Solartron 1260 frequency response analyzer over the frequency range from $1 \mathrm{MHz}$ to $0.1 \mathrm{~Hz}$ (24 points per decade) and a voltage perturbation amplitude of $20 \mathrm{mV}$ with zero DC bias. Data analysis was performed using the ZView2 commercial software package (Version 2.9b, Scribner Associates, Inc.).

Despite the loss of the majority of the samples due to $\mathrm{Pt}$ interconnectivity through the ceria films (i.e. short circuiting) several structures with appropriate electrical connectivity and robustness were prepared, and thus the measurements were successfully performed several $(>10)$ times for different films and/or electrodes. The essential results are captured in the behavior of two representative samples, the data from which are presented below. The physical characteristics of these films and experimental conditions under which they were examined are summarized in Table 2.

\section{Results}

\section{Microstructure}

The SEM images, Fig. 2, reveal that the desired columnar microstructure has been obtained, with good adhesion of the ceria film to the $200 \mathrm{~nm}$ thick Pt bottom electrode. The diameter of the columns is not clearly defined, but can be roughly specified as $\sim 100 \mathrm{~nm}$, based on the high contrast image presented in Fig. 2c. Individual grains comprising the columns appear to be even smaller, with an in-plane grain size of perhaps $30 \mathrm{~nm}$ or less. The X-ray diffraction results, Fig. 3, collected both before and after electrical characterization at $500{ }^{\circ} \mathrm{C}$, indicate that the films undergo negligible microstructural evolution over the course of the measurements. The data further reveal that the film has a strong (100) preferred orientation. The ratio of the integrated intensities of the (200) and

Table 2 Physical characteristics of films used for electrochemical measurements

\begin{tabular}{|c|c|c|c|}
\hline Film & Top electrode & Thickness $(\mu \mathrm{m})$ & Measurements \\
\hline 1 & Sputtered Pt (250 nm thick) & 3.6 & $p \mathrm{H}_{2} \mathrm{O}$ isobars (dry, 1.27 mbar, $8.73 \mathrm{mbar}$ ) \\
\hline 2 & Sputtered Pt (50 nm thick) & 5.9 & Isotherms $\left(250{ }^{\circ} \mathrm{C}, 300{ }^{\circ} \mathrm{C}, 350{ }^{\circ} \mathrm{C}\right)$ \\
\hline
\end{tabular}



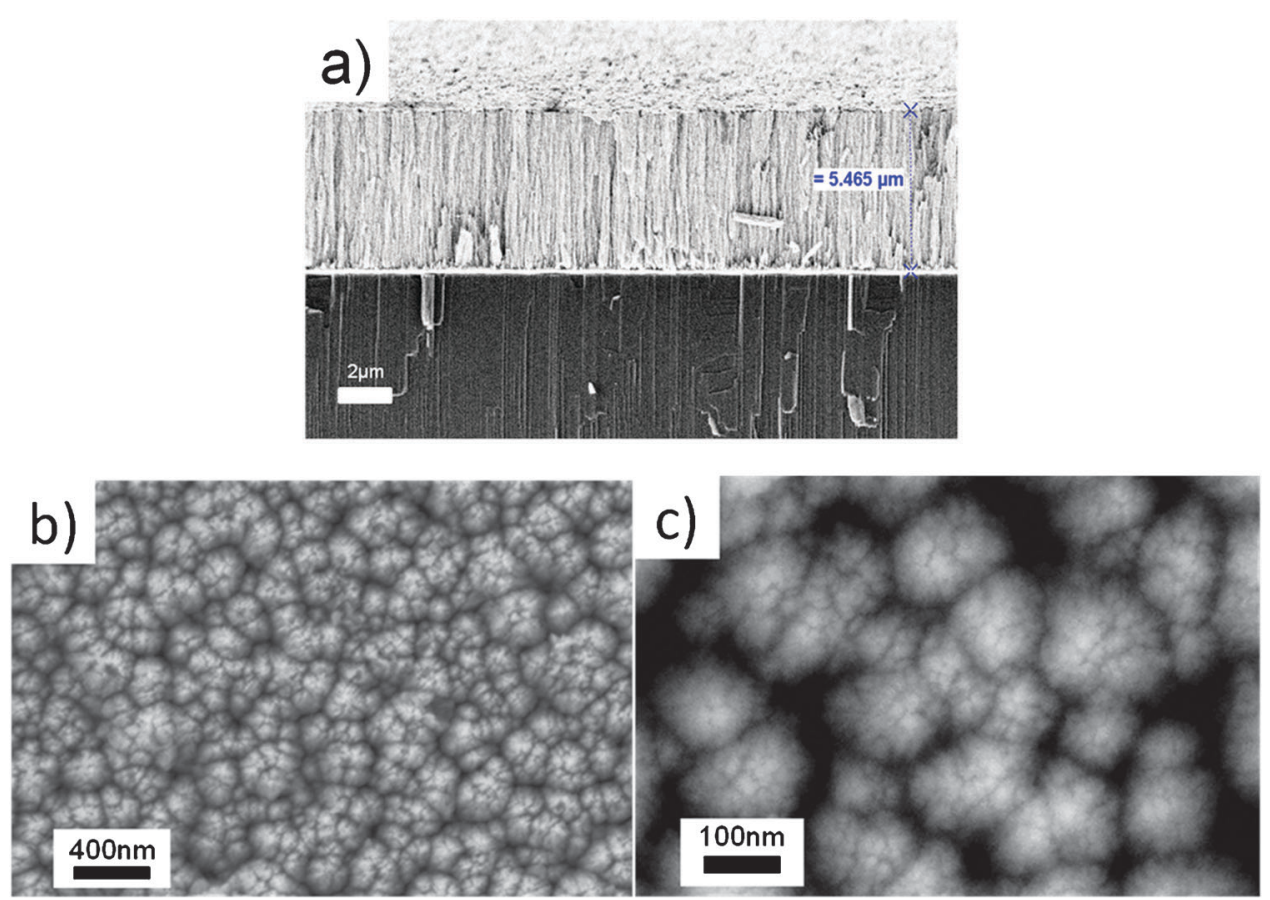

Fig. 2 Representative scanning electron microscopy (SEM) images of chemical vapor deposition (CVD) grown ceria on MgO(100). (a) Cross-sectional image (5 $5^{\circ}$ tilted) showing the columnar structure of the CVD ceria film. Bright boundary between the ceria film and the MgO substrate is the $200 \mathrm{~nm}$ thick Pt bottom electrode. (b) Planview image again revealing columnar features. (c) Higher magnification image of (b) in a high contrast mode revealing internal grain structure of the columns.

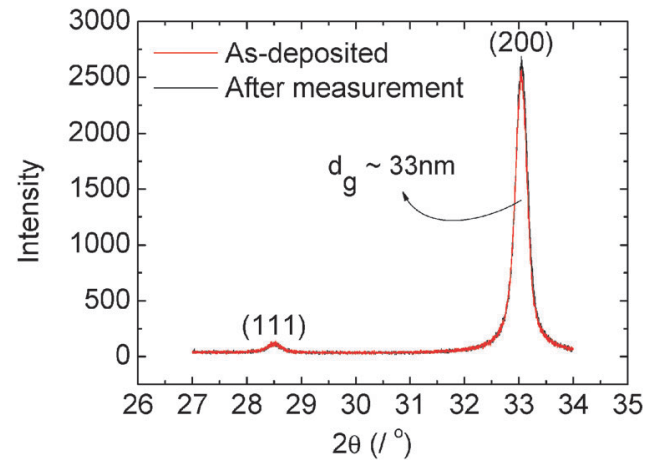

Fig. 3 Typical X-ray powder diffraction patterns from the ceria film ( $4.3 \mu \mathrm{m}$ thick in this case) grown on Pt coated $\mathrm{MgO}(100)$. Grain size $\left(d_{\mathrm{g}}\right)$ is calculated from FWHM of the 200 peak using the Scherrer formula after accounting for instrument broadening. There was no change in the X-ray pattern after exposure to the thermal conditions of electrical measurements.

(111) peaks, $\frac{I(200)}{I(111)}$, is about 32 in the film, which is many times greater than the expected value for polycrystalline ceria with random crystallite orientation, $\sim 0.3$. The out-of-the-plane grain size, based on the breadth of the (200) peak, is calculated to be $33 \mathrm{~nm}$.

\section{Electrical characterization}

Selected Nyquist plots $\left(Z_{\text {real }} v s . Z_{\text {imag }}\right.$ as parametric functions of frequency) are presented in Fig. 4 for a film exposed to three different gas atmospheres at a fixed temperature of $200{ }^{\circ} \mathrm{C}$. The spectra are qualitatively similar. All three display one high-frequency arc that extends to the origin and a second low-frequency response that, at the lowest frequencies (not shown), turns towards the real axis. The latter feature, which was found to vary in response to variations in the total gas flow rate, is attributed to the electrode behavior and is not considered further. With respect to the high-frequency response, in a polycrystalline ionic conductor the impedance spectra typically display, in contrast to the present results, distinct bulk and grain boundary arcs. ${ }^{10}$ On the other hand, it is not uncommon in the case of nanocrystalline ceria to observe a single, non-electrode response, ${ }^{11-14}$ as appears to be the case here. Grain boundary resistance in impurity-free ceria is due to the ion-blocking effect of space charge zones at the grain interfaces. Because the width of the space charge zone is inversely proportional to dopant concentration, the zone can extend in nominally undoped ceria over a distance of tens of nanometers. ${ }^{10}$ If the material is additionally nanostructured, the space charge zone can encompass the entire grain such that there is no distinction between bulk and grain boundary regions. Thus, the absence of a distinct grain boundary response in the present measurements is not inconsistent with the presence of grain boundaries normal to the direction of charge transport. The raw impedance data furthermore show that the overall resistance associated with this single response decreases dramatically with increasing water partial pressure.

The general shapes of the spectra suggest, at first glance, that the high frequency data are amenable to equivalent circuit modeling in terms of a single RQ subcircuit, where $\mathrm{R}$ is a resistor and $\mathrm{Q}$ is a constant phase element. ${ }^{15}$ Closer examination, however, reveals a change in the shape of the high frequency arc in response 
(a)

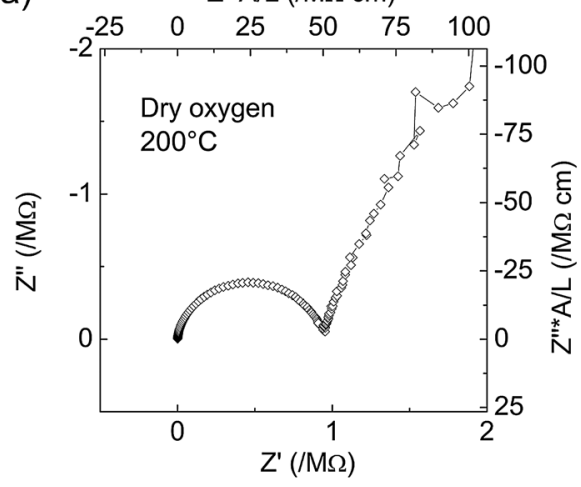

(b)

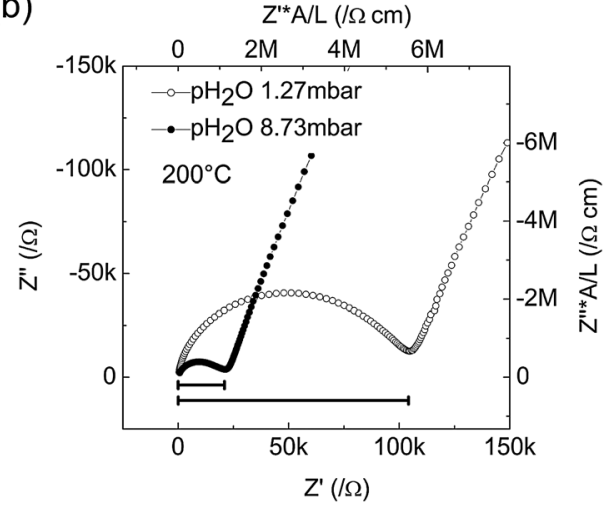

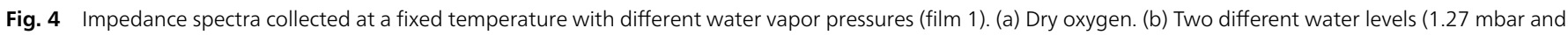
8.73 mbar). The effective DC resistivity, defined as $R_{\mathrm{M}}$, is simply taken as the $Z^{\prime}$ accompanying the minimum $-Z^{\prime \prime}$, as shown by the horizontal bars in (b).

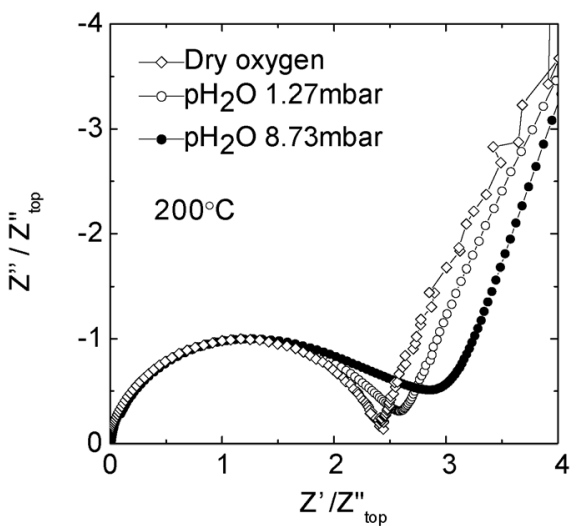

Fig. 5 Normalized representation of impedance spectra of Fig. 4 accentuating changes in the shape of the impedance arcs in response to changes in the atmosphere. Normalization is with respect to the maximum value of the imaginary component of impedance within the bulk response regime.

to changes in the gas atmosphere, suggesting the inadequacy of a simple RQ subcircuit to extract frequency independent properties. This behavior is underscored in Fig. 5 in which the three spectra of Fig. 4 are superimposed after normalization with respect to magnitude of the imaginary component of the impedance at the characteristic frequency (i.e. at the top of the arc). With increasing water partial pressure there is evidence of the emergence of an intermediate frequency feature that cannot be readily captured by simple equivalent circuit modeling. Thus, in order to avoid artifacts due to fitting according to an inappropriate equivalent circuit, the overall material resistance, $R_{\mathrm{M}}$, was defined as the value of the real impedance at the minimum in the imaginary impedance (as shown for example in Fig. 4b). The equivalent 'overall capacitance' is defined for completeness as $C_{\mathrm{M}}=1 / R_{\mathrm{M}} \omega_{0}$, where $\omega_{0}$ is the angular frequency at which the imaginary component of the impedance attains a maximum. The conductivity and relative dielectric constant are obtained by the usual transformation using film thickness and electrode area.

The capacitive and transport characteristics of the film are summarized in Fig. 6, with the relative dielectric constant presented in Fig. 6a and the conductivity in Fig. 6b. Whereas the dielectric constant is almost constant over the $T$ and $p \mathrm{H}_{2} \mathrm{O}$ range of the measurements, the conductivity varies by orders of magnitude. Furthermore, the relative dielectric constant, $\varepsilon_{\mathrm{r}}$, is similar to the bulk value as reported for dense, nearly singlecrystalline ceria. ${ }^{16}$ The slightly lower value measured here is attributed to the porosity of the films, Fig. 2 . If the difference is (a)

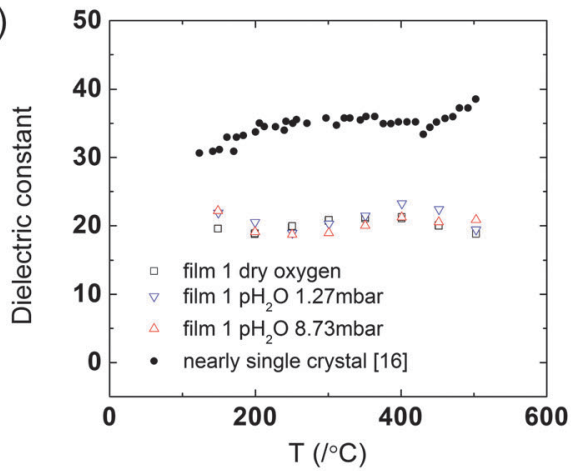

(b)

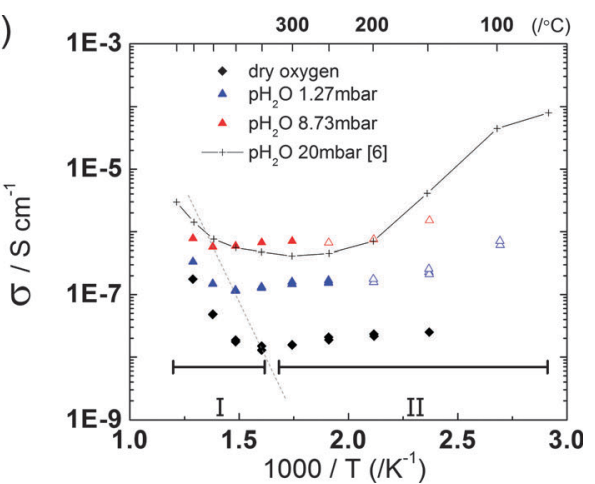

Fig. 6 Effective DC properties of film 1: (a) dielectric constant and (b) conductivity. The fully dense ceria dielectric constant is from the literature as measured under dry oxygen. Two regions are evident in the conductivity behavior. Region I: oxygen ion conduction is prevalent. Region II: proton conduction is prevalent. For comparison, proton conductivity reported for nanograined undoped ceria $\left(\mathrm{pH}_{2} \mathrm{O}=20 \mathrm{mbar}\right)$ is shown. ${ }^{6}$ 
attributed solely to such effects, the measured $\varepsilon_{\mathrm{r}}$ values imply a reasonable porosity of approximately $30 \%$ in the CVD grown film. Porous ceria films grown by pulsed laser deposition and with similar microstructural features ${ }^{17}$ have been found to display comparable levels of porosity. Rather significantly, the results in Fig. 6 show that the dielectric constant (being largely independent of the measurement conditions) is not correlated with the observed changes in the shape of the impedance spectra.

Turning to the results of Fig. 6b, the overall dependence of the conductivity on temperature, first decreasing then increasing with increasing temperature, with an inflection point at $\sim 400{ }^{\circ} \mathrm{C}$, and on $\mathrm{pH}_{2} \mathrm{O}$, is consistent with the identification of protons as the dominant mobile species at low temperature, and is in good agreement with typical behavior reported in the literature. ${ }^{6}$ Remarkably, the non-monotonic dependence of conductivity on temperature holds true even under nominally dry oxygen, at which proton sorption onto or incorporation into the oxide must be extremely limited. At higher temperatures oxygen ion conduction presumably dominates the transport process, as indicated by the observation of increasing conductivity with increasing temperature and a decreasing impact of water partial pressure. Moreover, with decreasing $p \mathrm{H}_{2} \mathrm{O}$ the inflection point in the conductivity as a function of temperature shifts to lower temperature, indicating that the temperature regime for oxygen ion conduction expands with decreasing chemical potential of water.

Ignoring for the moment the subtle change in the shape of the impedance response, the material can be roughly represented by an equivalent circuit comprised simply of a capacitor and a resistor in parallel. Given what is known about nanocrystalline ceria, we interpret the resistive element to correspond to proton migration along the surface of the parallel grain boundaries and/or open surfaces, and the dielectric element to the bulk. The insensitivity of the dielectric response to changes in $T$ and $p \mathrm{H}_{2} \mathrm{O}$, despite dramatic changes in the magnitude of the resistance and changes in the shape of the impedance response, is consistent with this interpretation.

The change in arc shape is reflected in the ratio of the arc diameter, $R_{\mathrm{M}}$, to its height, $-Z_{\text {top }}^{\prime \prime}$ (the value of the imaginary component impedance at the peak of the arc). This quantity attains a value of 2 for a perfect RC circuit and has magnitude greater than that when the arc is depressed due, for example, to the presence of a distribution of time constants ${ }^{18}$ and/or is composed of multiple overlapping arcs. A plot of this ratio as a function of $T$ and $p \mathrm{H}_{2} \mathrm{O}$, Fig. 7, shows that the deviation from 2 is greatest at intermediate temperatures with a peak value (approaching 3) that occurs at a temperature that shifts upwards with increasing water vapor pressure. These conditions roughly correspond to the conditions under which proton conductivity adopts an intermediate value-not the very high conductivity of low temperatures, but not the small proton conductivity at high temperatures at which oxide ion conduction begins to dominate the transport. Accordingly, we attribute the change in impedance features to the existence of a distribution of time constants under conditions of intermediate proton surface concentration. Such conditions can plausibly lead to a distribution of proton

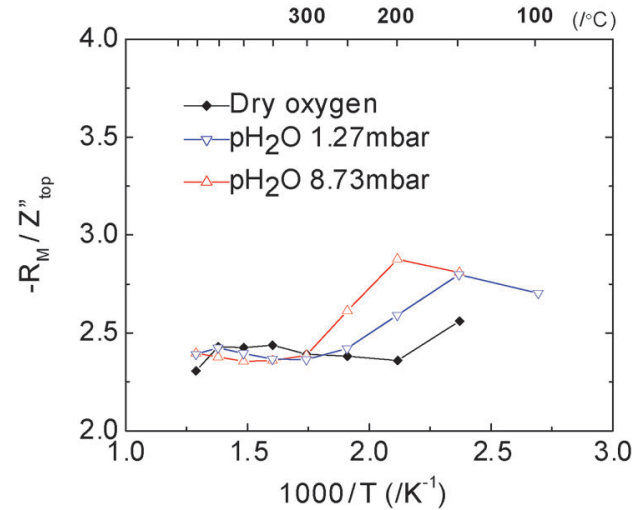

Fig. 7 The quantity $-R_{\mathrm{M}} / Z_{\text {top }}^{\prime \prime}$, shown here as a function of $T$ and $p \mathrm{H}_{2} \mathrm{O}$ for film 1 , provides a measure of the non-ideality of the impedance arc and reflects the breadth of the distribution of relaxation times. A value of 2 indicates an ideal, semi-circular impedance arc whereas larger values indicate an oblate shape. For all conditions at $T \geq 300{ }^{\circ} \mathrm{C}$, the arc remains relatively ideal. For $T<300{ }^{\circ} \mathrm{C}$, arc shape changes depending on the surrounding gas-phase water vapor pressure.

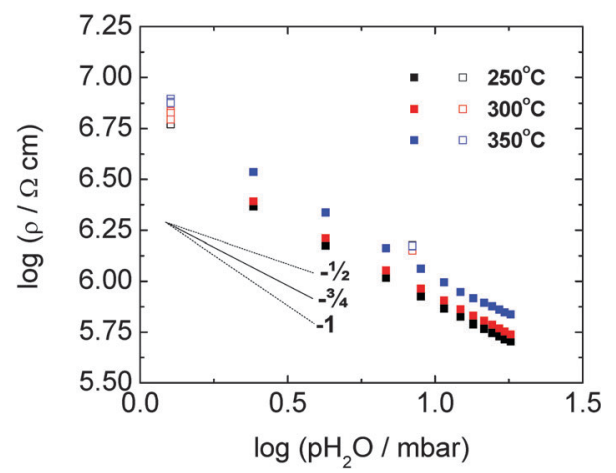

Fig. 8 Resistivity isotherms presented in double-logarithmic form. Open symbols: film 1. Closed symbols: film 2. For film 2, the fitted lines for isothermal data display slopes of $-0.758 \pm 0.006,-0.750 \pm 0.002$, and $-0.81 \pm 0.01$ at 250,300 , and $350{ }^{\circ} \mathrm{C}$, respectively.

environments as a result of the co-existence of highly and poorly hydrated regions.

A summary of the water partial pressure dependence of resistivity, as measured from both films 1 and 2, is presented in Fig. 8. The two samples display comparable resistivities for a given set of conditions, with slightly higher conductivity in film 1. The capacitance responses of the two films are also comparable (not shown). Film 2 has a slightly reduced volumetric capacitance, by $\sim 5 \%$, behavior attributed to slightly higher porosity. Differences in column surface morphology may explain the slight differences in resistivity. Under the conditions of these measurements the overall conductivity is large, and the ratio $-R_{\mathrm{M}} / Z_{\text {top }}^{\prime \prime}$ for film 2 ranged from 2.38 to 2.47 (comparable to the smallest values reported in Fig. 7), indicating a relatively ideal, single arc in the impedance response. The generally unchanging and almost ideal value of $-R_{\mathrm{M}} / Z_{\text {top }}^{\prime \prime}$ legitimizes a comparison of the resistivity as a function of purely environmental conditions and enabled impedance analysis by conventional equivalent circuit fitting. Specifically, the material behavior was represented 
by a parallel RQ subcircuit comprising a resistor of impedance $Z_{\mathrm{R}}=\mathrm{R}$ and a constant phase element of impedance $Z_{\mathrm{Q}}=$ $\left[Y(j \omega)^{n}\right]^{-1}$, where $\omega$ is the angular frequency, $j=\operatorname{sqrt}(-1)$, $Y$ and $n$ are constants, and $n$ ranges between 0 and 1 . Significantly, the conductivity measured for film 2 at 18 mbar $p \mathrm{H}_{2} \mathrm{O}$, the highest water partial pressure value examined, exceeds that of random nanocrystalline ceria measured at 20 mbar $\mathrm{pH}_{2} \mathrm{O}$ by a factor of 4 at $300{ }^{\circ} \mathrm{C}$. ${ }^{6}$ The result suggests that the aligned pore structure has provided measurable enhancement to the protonic conductivity.

The double logarithmic presentation of resistivity in Fig. 8 not only captures the decrease in $\rho$ with increasing $p \mathrm{H}_{2} \mathrm{O}$ already noted, but also shows the dependence to be exponential. The power law exponent was evaluated for film 2, examined at a relatively large number of water partial pressure values, and was found to be to $-0.758 \pm 0.006,-0.750 \pm 0.002$, and $-0.81 \pm 0.01$ at 250,300 and $350{ }^{\circ} \mathrm{C}$, respectively, in all cases rather close to $-\frac{3}{4}$. In an oxide in which proton uptake occurs in the bulk and oxygen vacancies are the predominant positive defects, the dependence of proton concentration on water vapor pressure can be predicted from the generic proton incorporation reaction:

$$
\mathrm{H}_{2} \mathrm{O}_{(\mathrm{g})}+V_{\mathrm{O}}^{\bullet \bullet}+\mathrm{O}_{\mathrm{O}}^{x}=2 \mathrm{OH}_{\mathrm{O}}^{\bullet}
$$

where the Kroger-Vink notation is utilized. ${ }^{19}$ Over a small change in proton concentration (the variation in conductivity recorded here is only about $10 \%$ ) and under the approximation of ideal solution behavior, eqn (1) implies that the proton resistivity (inversely proportional to concentration) should display a power law factor of $-\frac{1}{2}$, as opposed to the $-\frac{3}{4}$ observed here. It is noteworthy that a $-\frac{1}{2}$ dependence has, in fact, been previously reported for proton conducting Gd-doped ceria at $200{ }^{\circ} \mathrm{C}$ (grain size $\sim 108 \mathrm{~nm}$ ). ${ }^{20}$ There are many factors that could explain a deviation from a power law factor of $-\frac{1}{2}$ in undoped ceria even for bulk proton uptake, first amongst these being the low intrinsic oxygen vacancy concentration such that the effects of site saturation cannot be neglected. Nevertheless, the preponderance of the evidence suggests that the observed behavior reflects the characteristics of the surface absorption isotherm for $\mathrm{H}_{2} \mathrm{O}$ molecules on nanocolumnar ceria. Further experimentation, beyond the scope of the current work, is required to understand the origin of the $-\frac{3}{4}$ power law exponent.

\section{Summary and conclusion}

Through-plane AC impedance measurements were performed on MOCVD grown columnar ceria films. Proton conductivity was detected at temperatures below $350{ }^{\circ} \mathrm{C}$ regardless of gas phase water vapor pressure, as indicated by an increase in conductivity with decreasing temperature and increasing water vapor pressure. The capacitance, in all cases, is consistent in magnitude with the bulk dielectric response. Accordingly, the material is interpreted to display proton conductivity along the surfaces of the columnar grains, in parallel to bulk capacitance. Under conditions corresponding to intermediate proton conductivity
$\left(T=100\right.$ to $\left.250{ }^{\circ} \mathrm{C}\right)$ the impedance response changed from a somewhat ideal arc in the Nyquist representation to the one with detectable distortion. It is proposed that under such conditions proton surface uptake adopts an intermediate value relative to the available sites and hence a distribution of proton environments exists, leading to a distribution of relaxation times. In the most extreme cases (not shown), two distinct arcs emerged, suggesting two classes of relaxation processes. In the region of proton conductivity (high $\mathrm{pH}_{2} \mathrm{O}$ ), a power law dependence of conductivity on water vapor pressure was observed, with a power law exponent of $-\frac{3}{4}$. The origin of this behavior is unknown, but given the expectation of a power law exponent of $-\frac{1}{2}$ for bulk proton uptake, the result is taken to support the conclusion of surface proton conductivity. Grain boundary impedance at the interfaces between the grains forming the columns was not observed under any situation. It can be concluded that such interfacial resistance is either overwhelmed by the parallel proton transport or that overlapping space charge zones originating at the grain boundary interfaces imply no distinction between grain interior and grain boundary regions. It is not uncommon to observe a single-arc impedance response from nanostructured, undoped ceria, as reported here, and the result is most often attributed to the latter effect. Overall, the aligned pore structure of the ceria columnar films resulted in a factor of 4 higher proton conductivity than that of nanograined ceria sintered pellets ${ }^{6}$ examined under identical conditions.

\section{Acknowledgements}

The authors gratefully acknowledge financial support from the National Science Foundation under Grant No. CBET1038307. Dr. Yong Hao carried out all Pt depositions. Prof. Harry Atwater kindly provided access to DC magnetron sputtering instrumentation.

\section{References}

1 Z. Shao and S. M. Haile, Nature, 2004, 431, 170-173.

2 W. C. Chueh, C.-K. Yang, C. M. Garland, W. Lai and S. M. Haile, Phys. Chem. Chem. Phys., 2011, 13, 6442-6451.

3 N. Sakai, K. Yamaji, T. Horita, H. Yokokawa, Y. Hirata, S. Sameshima, Y. Nigara and J. Mizusaki, Solid State Ionics, 1999, 125, 325-331.

4 H. Yokokawa, T. Horita, N. Sakai, K. Yamaji, M. E. Brito, Y. P. Xiong and H. Kishimoto, Solid State Ionics, 2006, 177, 1705-1714.

5 H. J. Avila-Paredes, C.-T. Chen, S. Wang, R. A. De Souza, M. Martin, Z. Munir and S. Kim, J. Mater. Chem., 2010, 20, 10110-10112.

6 M. Shirpour, G. Gregori, R. Merkle and J. Maier, Phys. Chem. Chem. Phys., 2011, 13, 937-940.

7 T. Oh, Electrical, Electrochemical and Optical Characterization of Ceria Films, PhD thesis, California Inst. Technology, 2013.

8 I. M. Watson, M. P. Atwood, D. A. Cardwell and T. J. Cumberbatch, J. Mater. Chem., 1994, 4, 1393-1401. 
9 P. Scherrer, Göttinger Nachrichten, 1918, 2, 98-100.

10 X. Guo, W. Sigle and J. Maier, J. Am. Ceram. Soc., 2003, 86, 77-87.

11 H. Huang, T. M. Gür, Y. Saito and F. Prinz, Appl. Phys. Lett., 2006, 89, 143107.

12 D. Kek-Merl, J. Lappalainen and H. L. Tuller, J. Electrochem. Soc., 2006, 153, J15-J20.

13 E. Ballée, A. Ringuedé, M. Cassir, M. Putkonen and L. Niinistö, Chem. Mater., 2009, 21, 4614-4619.

14 J. H. Joo and G. M. Choi, J. Eur. Ceram. Soc., 2007, 27, 4273-4277.
15 B. A. Boukamp, Equivalent Circuit, University of Twente, The Netherlands, 1990.

16 T. C. Yeh, N. H. Perry and T. O. Mason, J. Am. Ceram. Soc., 2011, 94, 1073-1078.

17 W. Jung, J. O. Dereux, W. C. Chueh, Y. Hao and S. M. Haile, Energy Environ. Sci., 2012, 5, 8682-8689.

18 J. Fleig, Solid State Ionics, 2000, 131, 117-127.

19 Y. Yamazaki, P. Babilo and S. M. Haile, Chem. Mater., 2008, 20, 6352-6357.

20 E. Ruiz-Trejo and J. A. Kilner, J. Appl. Electrochem., 2009, 39, 523-528. 